\title{
Synthesis and stereochemistry of some multi methyl-substituted 1,3-dioxanes
}

\author{
Kalevi Pihlaja* and Jorma Mattinen \\ Department of Chemistry, University of Turku, FI-20014 Turku, Finland \\ E-mail: Kpihlaja@utu.fi
}

This paper is dedicated to the 60th birthday of Professor Ferenc Fülöp

\begin{abstract}
Several multimethyl-substituted 1,3-dioxanes [trans-2,4,4,6-tetramethyl (1), r-2,4,4,c-5,t-6pentamethyl- (2), r-2,4,4,t-5,t-6-pentamethyl (3) and trans-2,4,4,5,5,6-hexamethyl-1,3-dioxanes (4)] with 2,6-trans-disubstitution has been prepared via the Grignard reaction of the corresponding axial 2-methoxy-1,3-dioxanes. Inspection of their ${ }^{13} \mathrm{C} \mathrm{NMR}$ chemical shifts in respect of different substituent effects showed that $\mathbf{1}$ and $\mathbf{3}$ attain exclusively the 1,4-twist form whereas $\mathbf{2}$ and $\mathbf{4}$ still favor clearly the chair form due to the very strong steric interaction caused by the pseudo axial methyl groups at position 5. We also manage to equilibrate $\mathbf{1}$ and its cisepimer (5) although less than $1 \%$ of 4 was present at equilibrium. Thus only $-\Delta \mathrm{G}^{\mathrm{o}}=12.9 \pm 0.5 \mathrm{~kJ}$ $\mathrm{mol}^{-1}$ could be given and it compares well with some literature values. Since the conformational energy of 4-axial methyl group in 5 is $12.2 \mathrm{~kJ} \mathrm{~mol}^{-1}$ the $\Delta \mathrm{H}(1,4-\mathrm{CT})$ is equal to $25 \mathrm{~kJ} \mathrm{~mol}^{-1}$ again in good agreement with an earlier estimate.
\end{abstract}

Keywords: Multi-methyl 1,3-dioxanes, synthesis and stereochemistry, twist forms, ${ }^{13} \mathrm{C}$ NMR shift increments

\section{Introduction}

A great number of methyl-substituted 1,3-dioxanes have been prepared earlier. ${ }^{1}$ However, only after Eliel and Nader ${ }^{2}$ developed their special method for preparing trans-2,4,4,6-tetramethyl1,3-dioxane it became possible to synthesize also other trans-2,6-methyl-substituted 1,3dioxanes. We have been for a long time interested in the chair-twist equilibria of methylsubstituted 1,3-dioxanes. ${ }^{3-5}$ It was concluded quite some time ago that if there is no pseudo axial substituent in the twist form, the 2,4-syn-diaxially substituted derivatives attain a 2,5- or 1,4twist form (Figure 1) depending on the location of the geminal substitution in position 2 or 4 , respectively. ${ }^{3,6}$ The 1,4-twist form appears to be ca. $3 \mathrm{~kJ} \mathrm{~mol}^{-1}$ more stable than the 2,5-twist 
form $\left[\Delta \mathrm{H}(1,4-\mathrm{CT}) 25.0 \mathrm{~kJ} \mathrm{~mol}^{-1} ; \Delta \mathrm{H}(2,5-\mathrm{CT}) 28.7 \mathrm{~kJ} \mathrm{~mol}^{-1}\right]{ }^{3,5}$ By applying the method of Eliel and $\mathrm{Nader}^{2}$ we prepared a few trans-2,6-methyl-substituted derivatives where one or the other of these substituents occupies an axial orientation (if being in a chair form) to get further insight into the chair-twist problem.

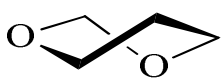

1,4-Twist

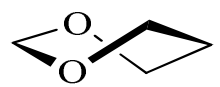

2,5-Twist

Figure 1. The two different twist forms for 1,3-dioxane.

\section{Results and Discussion}

The experimental ${ }^{13} \mathrm{C}$ NMR chemical shifts for the compounds $\mathbf{1 - 4}$ are given in Table 1 together with those estimated for $\mathbf{2 , 3}$ and $\mathbf{4}$ using the shift increments reported earlier by Pihlaja et al. $^{3-5}$

$$
\delta \mathrm{C}(\mathrm{x})=\delta \mathrm{C}_{\mathrm{p}}(\mathrm{x})+\Sigma \mathrm{SE}(\mathrm{x})
$$

In this equation $\delta \mathrm{C}(\mathrm{x})$ is the $\mathrm{C}-13$ chemical shift of the $\mathrm{xth}$ carbon, $\delta \mathrm{C}_{\mathrm{p}}(\mathrm{x})$ the shift of this carbon in the parent compound and $\Sigma \mathrm{SE}(\mathrm{x})$ the sum of substituent effects influencing on the $\mathrm{xth}$ carbon.

It has already been shown that $\mathbf{1}$ attains the 1,4-twist form ${ }^{2,3}$ and by comparing its chemical shifts with those of $\mathbf{3}$ it is easy to believe that also $\mathbf{3}$ is predominantly in the 1,4-twist form (Fig. 2) since 2-Me, 6-Me and 5-Me attain there pseudo equatorial positions and both methyl groups at C-4 are isoclinal thus being able to avoid any major interactions. Table 1 lists the chemical shifts estimated for $\mathbf{3}$ by adding to the chemical shifts of $\mathbf{1}$ additional increments based on the orientation of the 5-methyl substituent (pseudo-equatorial) in the 1,4-twist form in relation to the other substituents. Despite the fact that the additional increments were originally derived for the chair form the very good agreement between the calculated and estimated chemical shifts (Table 1) proves that 3 favors greatly the 1,4-twist form. The $J_{\mathrm{H}-5, \mathrm{H}-6}=10.4 \mathrm{~Hz}$ fits also very well for this 1,4-twist structure where both $\mathrm{H}-5$ and H-6 are pseudoaxial. In fact the sum of $J_{\mathrm{H}-4, \mathrm{H}-5}$ couplings in the 1,4-twist form of 1 is $15 \mathrm{~Hz}\left(2 \times 7.5 \mathrm{~Hz}^{8}\right)$ corresponding roughly an average of 5 and $10 \mathrm{~Hz}$.

The increments published by Pihlaja et $a l^{3-5}$ did not include those for $4 a 5 e 6 a$ - and $4 a 5 a 6 a$ methyl substitutions. In fact compound 2 (Fig. 3) which definitively exist in a deformed chair form $^{1}$ (since it has a pseudo axial methyl group at C-4 in the 2,5-twist form and a pseudo axial methyl group at C-5 in the 1,4-twist form) can be applied to derive the $4 \mathrm{a} 5 \mathrm{e} 6 \mathrm{a}$ increment. The ${ }^{13} \mathrm{C}$ chemical shifts evaluated for the alternative $2 \mathrm{a}, 4,4,5 \mathrm{a}, 6 \mathrm{e}-\mathrm{Me}_{5}$ conformation indicate that 2 must exist practically in the $2 \mathrm{e}, 4,4,5 \mathrm{e}, 6 \mathrm{a}-\mathrm{Me}_{5}$ chair only. In this case the value of the $J_{\mathrm{H}-5, \mathrm{H}-6}=$ $5.6 \mathrm{~Hz}$ is close to this value $(4.9 \mathrm{~Hz})$ found for $2,2,4,4,5 \mathrm{e}-\mathrm{Me}$-pentamethyl-1,3-dioxane ${ }^{3}$ and fits 
nicely within the ranges $4.6-6.0 \mathrm{~Hz}^{7 \mathrm{a}}$ and $5.0-6.6 \mathrm{~Hz}^{7 \mathrm{~b}, \mathrm{c}}$ reported earlier for the $J_{5 \mathrm{ax}, 6 \mathrm{eq}}$ type couplings in the chair conformations of methyl-substituted 1,3-dioxanes.

Table 1. Observed and calculated ${ }^{13} \mathrm{C}$ NMR chemical shifts $(\delta)$ for the synthesized multi methylsubstituted 1,3-dioxanes (1-4). For the parent compound $\delta(\mathrm{C}-2)=94.29, \delta(\mathrm{C} 4 / 6)=66.92$ and $\delta(\mathrm{C}-5)=26.56 \mathrm{ppm}$

\begin{tabular}{|c|c|c|c|c|c|c|c|}
\hline Carbon & & $\mathbf{1}^{1}$ & $2^{7,10}$ & $\mathbf{3}^{1,9}$ & $4^{8}$ & $\begin{array}{l}\text { trans-2,4,4, } \\
5,6,6-\mathrm{Me}_{6}\end{array}$ & $\begin{array}{l}\text { cis- } \\
2,4,4,5 \\
6,6-\mathrm{Me}_{6}\end{array}$ \\
\hline \multirow[t]{4}{*}{$\mathrm{C}-2$} & Obsd & 88.81 & 85.89 & 88.16 & 87.39 & 86.25 & 88.04 \\
\hline & Calcd & & $85.89^{2, \mathrm{a}}$ & $88.48^{4, b}$ & 87.39 & & \\
\hline & Calcd & & $94.86^{3}$ & & $84.06^{5, \mathrm{a}}$ & & \\
\hline & Calcd & & & & $94.48^{6}$ & & \\
\hline \multirow[t]{4}{*}{ C-4 } & Obsd & 71.92 & 72.28 & 75.49 & 76.91 & 74.68 & 75.37 \\
\hline & Calcd & & $72.13^{2, \mathrm{a}}$ & $75.34^{4, c}$ & 76.91 & & \\
\hline & Calcd & & $72.80^{3}$ & & $77.24^{5, \mathrm{a}}$ & & \\
\hline & Calcd & & & & $76.21^{6}$ & & \\
\hline \multirow[t]{4}{*}{$C-5$} & Obsd & 41.51 & 40.53 & 42.56 & 37.75 & 46.66 & 44.87 \\
\hline & Calcd & & $40.53^{2, \mathrm{a}}$ & $43.18^{4, \mathrm{~d}}$ & 37.76 & & \\
\hline & Calcd & & $39.34^{3}$ & & $37.95^{5, \mathrm{a}}$ & & \\
\hline & Calcd & & & & $37.35^{6}$ & & \\
\hline \multirow[t]{4}{*}{$C-6$} & Obsd & 65.38 & 71.69 & 70.82 & 76.63 & 74.68 & 75.37 \\
\hline & Calcd $^{2}$ & & $71.53^{2, \mathrm{a}}$ & $70.54^{4, \mathrm{e}}$ & 76.59 & & \\
\hline & Calcd $^{3}$ & & $63.04^{3}$ & & $80.76^{5, a}$ & & \\
\hline & Calcd & & & & $67.72^{6}$ & & \\
\hline \multirow[t]{2}{*}{$\mathrm{C}-\mathrm{Me}$} & Obsd & 28.9,27.7, & $31.0,21,8$, & $26.8,23.2$ & $26.7,25.8$ & 31.7,22.0, & 28.9,25.1, \\
\hline & & $21.8,21.7$ & $\begin{array}{l}20.8,14.5 \\
12.8\end{array}$ & $\begin{array}{l}22.0,19.6, \\
13.2\end{array}$ & $\begin{array}{l}23.5,21.7 \\
20.7,16.2\end{array}$ & $19.9,12.1$ & $22.7,12.6$ \\
\hline
\end{tabular}

${ }^{1}$ These compounds attain the 1,4-twist form. ${ }^{2}$ These values calculated for the $2 e 5 e 6 a$-Me $\mathrm{M}_{3}$ chair. ${ }^{3}$ These values calculated for the $2 a 5 a 6 e-\mathrm{Me}_{3}$ chair. ${ }^{4}$ These values calculated for the 1,4-twist form. ${ }^{5}$ These values calculated for the $2 e 6 a-\mathrm{Me}_{2}$ chair. ${ }^{6}$ These values calculated for the $2 a 6 e-\mathrm{Me}_{2}$ chair. ${ }^{7}$ This compound attains predominantly the $2 \mathrm{e} 5 \mathrm{e} 6 \mathrm{a}$ chair form. ${ }^{8}$ This compound is a $68: 32$ mixture of $2 e 6 a$ and $2 a 6 e$ chair forms. ${ }^{a}$ These values already include the $4 \mathrm{a} 5 \mathrm{e} 6 \mathrm{a}$ increments listed in Table 2. ${ }^{\mathrm{b}} 88.81-0.33$ ppm. ${ }^{1}{ }^{\mathrm{c}} 71.92+6.34-0.30-0.55-0.59-1.48 \quad \mathrm{ppm} .{ }^{1}$ ${ }^{\mathrm{d}} 41.51+3.59-2(0.61)-0.70 \mathrm{ppm}^{1}{ }^{1} \mathrm{e}^{6} 65.38+6.34-0.30-0.55-1.12+0.79 \mathrm{ppm}^{1}{ }^{1}{ }^{9} J_{\mathrm{H}-5, \mathrm{H}-6}=10.4 \mathrm{~Hz}$. ${ }^{10} J_{\mathrm{H}-5, \mathrm{H}-6}=5.6 \mathrm{~Hz}$. 


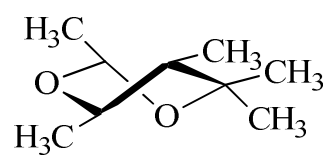

3

Figure 2. 1,4-Twist form of $r$-2,4,4,c-5,t-6-pentamethyl-1,3-dioxane.

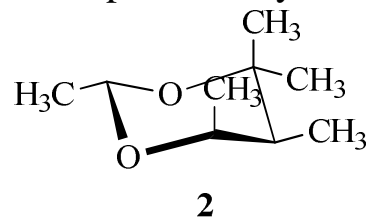

Figure 3. The 2e6a-chair form of $r-2,4,4, t-5, t-6$-pentamethyl-1,3-dioxane.

Table 2 shows the 4a5e6a increments at each ring carbon atom. The calculated shifts given in Table 1 for the 2e6a chair form already include the 4a5e6a increments. To obtain the 4a5a6a increment we need a bit more complicated approach. Trans-2,4,4,5,5,6-hexamethyl-1,3-dioxane also exist in a deformed chair form ${ }^{3}$ since it would have pseudo axial methyl groups ${ }^{3}$ both in the 1,4- (one of the 5-methyls) and 2,5-twist forms (one of the 4-methyls). We can estimate the C-13 chemical shifts for the possible chair conformations (2e,4,4,5,5,6a and 2a,4,4,5,5,6e; Fig. 4) using the equations

$$
\begin{aligned}
& \delta_{\text {obs }}(\mathrm{C}-4)=\mathrm{x}\left[\delta_{\text {est }}^{1}(\mathrm{C}-4)+\mathrm{y}\right]+(1-\mathrm{x}) \delta_{\text {est }}^{2}(\mathrm{C}-4) \\
& \delta_{\text {obs }}(\mathrm{C}-6)=\mathrm{x}\left[\delta_{\text {est }}^{1}(\mathrm{C}-6)+\mathrm{y}\right]+(1-\mathrm{x}) \delta_{\text {est }}^{2}(\mathrm{C}-6) \\
& 76.91=x[73.44+y]+(1-x) 76.21 ; \delta_{\text {est }}(C-4)=76.91 \mathrm{ppm} \\
& 76.63=x[76.96+y]+(1-x) 67.72 ; \delta_{\text {est }}(C-6)=76.59 \mathrm{ppm}
\end{aligned}
$$

which gives $\mathrm{x}=0.68$ and $\mathrm{y}=4 \mathrm{a} 5 \mathrm{a} 6 \mathrm{a}-$ increment $=3.80 \mathrm{ppm}$ at $\mathrm{C}-4 / 6$. The corresponding increments are then easy to derive for C-2 and C-5. For C-2

$$
\begin{aligned}
\delta_{\text {obs }}(\mathrm{C}-2) & \left.=0.68\left[\delta_{\text {est }}^{1} \mathrm{C}-2\right)+\mathrm{y}\right]+0.32 \delta^{2}{ }_{\text {est }}(\mathrm{C}-2) \\
87.39 & =0.68[86.12+\mathrm{y}]+0.32(94.38)
\end{aligned}
$$

which gives $\mathrm{y}=4 \mathrm{a} 5 \mathrm{a} 6 \mathrm{a}-\mathrm{increment}=-2.06 \mathrm{ppm}$.

Correspondingly,

$$
\begin{aligned}
& \left.\delta_{\text {obs }}(\mathrm{C}-5)=0.68\left[\delta_{\text {est }}^{1} \mathrm{C}-5\right)+\mathrm{y}\right]+0.32 \delta_{\text {est }}^{2}(\mathrm{C}-5) \\
& 37.75=0.68[37.53+\mathrm{y}]+0.32(37.35)
\end{aligned}
$$

which gives $\mathrm{y}=4 \mathrm{a} 5 \mathrm{a} 6 \mathrm{a}$-increment $=1.07 \mathrm{ppm}$. Now it is possible to estimate also the $4,4,5 \mathrm{e}, 6,6$ and 4,4,5a,6,6-increments based on the chemical shifts of trans- and cis-2,4,4,5,6,6-hexamethyl1,3-dioxanes determined earlier ${ }^{4}$ and listed in Table 2 . The values of the derived increments are given in Table 2 and their values indicate clearly that these compounds could not be applied to derive the 4a5e6a- and 4a5a6a-increments due to further effects caused by the 4,4,5e,6,6- and 4,4,5a,6,6-substitutions (Table 2). 
The equilibration of $\mathbf{1}$ and $\mathbf{5}$ (Fig. 5) pointed out that cis-2,4,4,6-tetramethyl-1,3-dioxane $\mathbf{5}$ is clearly more stable than $\mathbf{1}$ which attains the 1,4-twist form. ${ }^{2,3}$ Although we carried out the equilibrations at three temperatures (Table 3) the amount of $\mathbf{1}$ at equilibrium with $\mathbf{5}$ was so small that the integration of its peak ought to be carried out manually which caused a substantial deviation (Table 3) and therefore we just accept the average $-\Delta \mathrm{G}^{\mathrm{o}}=12.9 \mathrm{~kJ} \mathrm{~mol}^{-1}$ to represent the standard Gibbs energy difference between 5 (having a 4-axial methyl, the conformational energy ${ }^{3}$ of which is $12.2 \mathrm{~kJ} \mathrm{~mol}^{-1}$ ) giving a value ca. $25 \mathrm{~kJ} \mathrm{~mol}^{-1}$ for $\Delta \mathrm{H}(1,4-\mathrm{CT})$. The former value $\left(-12.9 \mathrm{~kJ} \mathrm{~mol}^{-1}\right)$ is in good agreement with the calculations of Burkert ${ }^{6}$ based on molecular mechanical computations but is far from the estimate $-\Delta \mathrm{G}^{0} \leq 22.8 \mathrm{~kJ} \mathrm{~mol}^{-1}$ given by Eliel and Nader. ${ }^{2}$ The value $25 \mathrm{~kJ} \mathrm{~mol}^{-1}$ has been reported for $\Delta \mathrm{H}(1,4-\mathrm{CT})$ also earlier. ${ }^{3}$

Table 2. ${ }^{13} \mathrm{C}$ NMR shift effects caused by the $4 a 5 e 6 a-\mathrm{Me}_{3}$ and $4 a 5 a 6 a-\mathrm{Me}_{3}$ substitutions

\begin{tabular}{ccccc}
\hline Substitution & \multicolumn{5}{c}{ Shift Effects, ppm } \\
\hline $4 a 5 e 6 a$ & C-2 & C-4 & C-5 & C-6 \\
$4 a 5 a 6 a$ & 0.57 & -0.40 & 0.29 & -0.40 \\
$4,4,5 \mathrm{e}, 6,6$ & -2.06 & 3.80 & 1.07 & 3.80 \\
$4,4,5 \mathrm{a}, 6,6$ & -0.58 & 1.19 & - & 1.19 \\
\hline
\end{tabular}

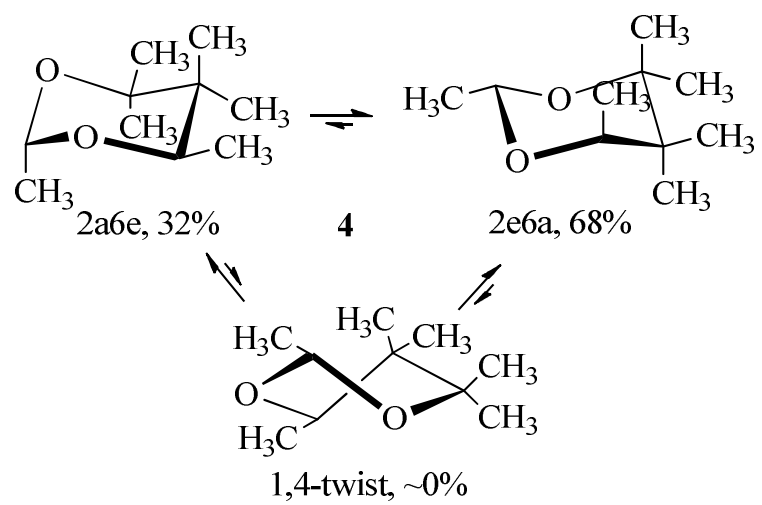

Figure 4. Conformational equilibrium for trans-2,4,4,5,5,6-hexamethyl-1,3-dioxane.

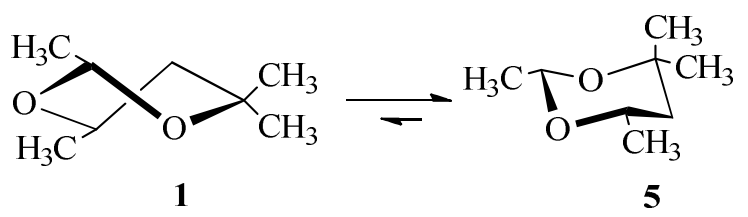

Figure 5. The equilibrium between the 1,4-twist form of trans- and the chair form of cis-2,4,4,6tetramethyl-1,3-dioxanes. 
Table 3. Equilibrium constants and standard Gibbs energy differences between cis- $\mathbf{5}$ and trans2,4,4,6-tetramethyl-1,3-dioxanes 1 at three temperatures

\begin{tabular}{llll}
\hline $\mathrm{T}, \mathrm{K}$ & \%trans & $K=[\mathbf{5}] /[\mathbf{1}]$ & $-\Delta \mathrm{G}, \mathrm{kJ} \mathrm{mol}^{-1}$ \\
\hline 298 & $0.65 \pm 0.1$ & $153 \pm 30$ & $12.5 \pm 0.5$ \\
313 & $0.7 \pm 0.1$ & $142 \pm 30$ & $12.9 \pm 0.5$ \\
333 & $0.8 \pm 0.15$ & $120 \pm 25$ & $13.4 \pm 0.6$ \\
\hline
\end{tabular}

Av. $12.9 \pm 0.5 \mathrm{~kJ} \mathrm{~mol}^{-1}$

\section{Conclusions}

Multi methyl-substituted 1,3-dioxanes with trans-2,6-methyl groups favor deformed chair forms to avoid pseudo axial groups at C-4- or C-5 in the 1,4- or 2,5-twist forms. If, however, the twist forms contain only pseudo equatorial or isoclinal methyl substituents the compounds prefer usually 1,4-twist form.

\section{Experimental Section}

General. 2-Methyl-2,4-pentanediol (Fluka AG, purum) was distilled before use. Trimethyl orthoformate was also from Fluka AG (purum) and it was also purified by distillation.

2,3-Dimethyl-2,4-pentanediol was prepared from ethyl-3.hydroxy-2-methyl butanoate ${ }^{9}$ as reported earlier. ${ }^{10}$ B.p. $385-390 \mathrm{~K}$ at $1 \mathrm{kPa}$ and $\mathrm{n}_{\mathrm{D}}{ }^{298} 1.4465$ (Lit. ${ }^{10}$ b.p. $381-385 \mathrm{~K}$ at $0.9 \mathrm{kPa}$ and $\mathrm{n}_{\mathrm{D}}{ }^{293} 1.4478$. Yield $49 \%$.

2,3,3-trimethyl-2,4-pentanediol was prepared from ethyl-3-hydroxy-2,2-dimethyl butanoate ${ }^{9}$ as reported earlier. ${ }^{10}$ M.p. $357 \mathrm{~K}$ (Lit. ${ }^{10} 360 \mathrm{~K}$ ). Yield $78 \%$.

Preparation of 2-methoxy-1,3-dioxanes. A $250 \mathrm{ml}$ three-necked bottle was equipped with a magnetic stirrer, a heating mantel and a distillation system. $0.3 \mathrm{~mol}$ of trimethyl orthoformate together with an equivalent amount of an 1,3-diol was placed in the bottle. $60 \mathrm{ml}$ of cyclohexane (Merck, reinst) was purified by distillation and added in the bottle together with a catalytic amount of $p$-toluenesulfonic acid (Merck, pa). Thereafter the mixture was heated whereupon the atseotropic mixture formed by the methanol product and cyclohexane was distilled off. The heating was continued until the vapor reached a $353 \mathrm{~K}$ temperature. At this stage the reaction mixture was allowed to cool to room temperature. Then $1-2 \mathrm{~g}$ of $\mathrm{K}_{2} \mathrm{CO}_{3}$ was added and the mixing was continued for two hours to neutralize the catalytic acid. Then the mixture was filtrated with mild suction. The precipitate was washed three times with ether and the solvent 
was removed from the combined liquid phase by evaporation at ordinary pressure. The product was distilled under reduced pressure through a short Vigreux column. The raw product boiled within a range of 12-15 degrees. The isomeric products (when possible) were separated with a Perkin Elmer 251 Auto Annular Still precision distiller at reduced pressure. The distillate was collected on anhydrous $\mathrm{K}_{2} \mathrm{CO}_{3}$ to avoid epimerization. 2-Methoxy-4,4,5,6-tetramethyl-1,3dioxane decomposed in the precision distiller. A $90 \%$ pure product was obtained by distilling it through a Hemppel column equipped with a vacuum mantle.

${ }^{13}$ C NMR spectra. The noise-decoupled spectra were recorded on a Jeol GX-400 spectrometer operating at $100.53 \mathrm{MHz}$ for ${ }^{13} \mathrm{C}$ ( and $399.78 \mathrm{MHz}$ for ${ }^{1} \mathrm{H}$ ). All spectra were recorded in $5 \mathrm{~mm}$ o.d. tubes using the solvent $\left(\mathrm{CDCl}_{3}\right)$ deuterium signal for field locking. Internal TMS was used as the reference.

Starting materials and their physical constants. Trans-2-Methoxy-4,4,6-trimethyl-1,3-dioxane was prepared from 2-methyl-2,4-pentanediol and trimethyl orthoformate. ${ }^{2,3}$ B.p. $328-330 \mathrm{~K}$ at $2.1 \mathrm{kPa}$. It was identified based on its ${ }^{13} \mathrm{C}$ NMR spectrum (Table 4). Yield $63 \%$.

$r$-2-MeO,4,4,c-5,t-6-Tetramethyl and $r$-2-MeO,4,4,t-5,t-6-tetramethyl-1,3-dioxanes were prepared from 2,3-dimethyl-3-hydroxy-2,4-pentanediol and trimethyl orthoformate. B.p. 347-356 $\mathrm{K}$ at $3.3 \mathrm{kPa}$. The product consisted of all four possible isomers of which only the ${ }^{13} \mathrm{C} \mathrm{NMR}$ spectrum (Table 4 ) of the main product $r-2-\mathrm{MeO}, 4,4, c-5, t-6-\mathrm{Me}_{4}$ could be solved. Yield $33 \%$.

Table 4. Observed ${ }^{13} \mathrm{C}$ NMR chemical shifts $(\delta)$ of the prepared 2-methoxy-1,3-dioxanes

\begin{tabular}{cccc}
\hline Carbon & $t$-2-OMe, $4,4,6-\mathrm{Me}_{3}$ & $t$-2-OMe, $4,4,5,5,6-\mathrm{Me}_{5}$ & $r-2-\mathrm{OMe}, 4,4, c-5, t-6-\mathrm{Me}_{4}{ }^{1}$ \\
\hline $\mathrm{C}-2$ & 113.2 & 107.6 & 107.6 \\
$\mathrm{C}-4$ & 76.5 & 79.3 & 72.5 \\
$\mathrm{C} .5$ & 49.0 & 37.3 & 44.5 \\
$\mathrm{C}-6$ & 64.9 & 74.1 & 69.5 \\
$\mathrm{C}-\mathrm{OMe}$ & 49.1 & 51.8 & 52.3 \\
$\mathrm{C}-\mathrm{Me}$ & $26.0,23.8,23.2$ & $24.8,21.1,20.3,15.55,15.2$ & $29.1,19.4,18.8,11.2$ \\
\hline
\end{tabular}

${ }^{1}$ This compound included also some amount of the $r$-2-OMe,4,4,t-5,t-6-Me $\mathrm{Me}_{4}$ epimer.

Trans-2-MeO,4,4,5,5,6-pentamethyl derivative (Table 4) was prepared from 2,3,3-trimethyl-2,4pentanediol and trimethyl orthoformate. B.p. $372-373$ at $3.3 \mathrm{kPa}$. Yield $63 \%$.

Grignard reaction with 2-methoxy-1,3-dioxanes. Methyl magnesium iodide was prepared by adding a 1:1 mixture of absolute ether and methyl iodide in a mixture containing magnesium chips, a small amount of absolute ether and a few crystals of iodine. Addition was done within an hour with such a rate that a smooth reflux occurred. Thereafter the mixture was stirred for another 15 minutes. A 1:1 mixture of absolute ether and a 2-methoxy-1,3-dioxane were added dropwise in the Grignard reagent. The reaction mixture was warmed until the main reaction 
began. The mixture was refluxed for another hour after addition of all of methoxy compound. The reflux was ceased when the color of the Grignard reagent vanished and a dark oily precipitate formed. The reaction mixture was allowed to cool to room temperature before ca. 3.5 $\mathrm{ml}$ of ice cold saturated ammonium chloride solution was added with vigorous stirring. The white precipitate formed was separated with a mild suction and washed with six $50 \mathrm{ml}$ portions of warm ether. The combined ether extracts were dried with anhydrous $\mathrm{MgSO}_{4}$ and excess ether evaporated with rotavapor and the rest by distillation under normal pressure. The product was fractionated at reduced pressure and collected on anhydrous $\mathrm{K}_{2} \mathrm{CO}_{3}$ to avoid epimerization.

\section{Multi methyl-substituted 1,3-dioxanes preparation}

Trans-2,4,4,6-tetramethyl-1,3-dioxane 1 (including some $10 \%$ of the cis-isomer 5) was prepared from trans-2-methoxy,4,4,6-trimethyl-1,3-dioxane (0.03 mol of the methoxy compound, 0.03 mol of $\mathrm{Mg}$ and $0.03 \mathrm{~mol}$ of methyl iodide). B.p. $327-328 \mathrm{~K}$ at $3.7 \mathrm{kPa} .{ }^{2}$ Yield $31 \%$. Its ${ }^{13} \mathrm{C}$ spectrum is given in Table $1 . \mathrm{C}_{8} \mathrm{H}_{18} \mathrm{O}_{2}$ : Calcd C 66.63\%, $\mathrm{H} 11.18 \%$, O 22.19\%; Obsd 66.45\% $11.26 \%, 22.37 \%$ (as the difference).

$\boldsymbol{r}$-2,4,4,c-5,t-6-Pentamethyl- 3 and $\boldsymbol{r}$-2,4,4,t-5,t-6-pentamethyl-1,3-dioxanes 2 were prepared from a mixture of $r$-2-MeO,4,4,c-5,t-6-tetramethyl and $r$-2-MeO,4,4,t-5,t-6-tetramethyl-1,3dioxanes $(0.03 \mathrm{~mol}$ of the mixture of the methoxy compounds, $0.03 \mathrm{~mol} \mathrm{of} \mathrm{Mg}$ and $0.3 \mathrm{~mol}$ of methyl iodide). B.p. of products $329-330 \mathrm{~K}$ at $2.0 \mathrm{kPa}$. The products were identified based on their ${ }^{13} \mathrm{C}$ NMR spectra (Table 1). $\mathrm{C}_{9} \mathrm{H}_{20} \mathrm{O}_{2}$ : Calcd C $68.31 \%, \mathrm{H} 11.46 \%$, O 20.22\%; Obsd $68.45 \% 11.36 \%, 20.19$ (as the difference).

trans-2,4,4,5,5,6-Hexamethyl-1,3-dioxane (4) was prepared from trans-2-MeO,4,4,5,5,6-pentamethyl-1,3-dioxane (0.03 mol of the methoxy compound, $0.03 \mathrm{~mol}$ of $\mathrm{Mg}$ and $0.3 \mathrm{~mol}$ of methyl iodide). B.p. $351-353 \mathrm{~K}$ at $2.3 \mathrm{kPa}$. Yield $62 \%$. The product was identified based on its ${ }^{13} \mathrm{C} \mathrm{NMR}$ spectrum (Table 1). $\mathrm{C}_{10} \mathrm{H}_{20} \mathrm{O}_{2}$ : Calcd 69.78\%, H 11.63\%, O 18.53\%; Obsd 69.60\%, H 11.72\%, $18.68 \%$ (as the difference).

\section{Equilibration of trans-2,4,4,6-tetramethyl-1,3-dioxane 1 with its epimer}

It was found out that $\mathbf{1}$ consisted of ca. $10 \%$ of its epimer cis-2,4,4,6-tetramethyl-1,3-dioxane $\mathbf{5}$. It has been shown earlier that $\mathbf{1}$ attains the 1,4-twist form and $\mathbf{5}$ exists in the chair form. ${ }^{3,4}$ The peaks of both isomers were well separated in gas chromatogram and therefore this technique was applied for the analysis of their equilibrium mixtures (Fig. 5) at three different temperatures. The equilibration was carried out in ether solution which was 0.1 molar in respect of both the substrate and the catalyst, trifluoroacetic acid (EGA Chemie, purum). The samples were sealed in glass ampoules and equilibration was carried out at 298, 313 and $333 \mathrm{~K}$. The equilibrium mixtures were analyzed on a Perkin Elmer Sigma 2 B gas chromatograph using a $30 \mathrm{~m}$ XE 60 capillary column. The samples were neutralized before analysis with triethyl amine (Fluka AG, purum). The results of equilibrations after 100 days are shown in Table 4. 


\section{References}

1. (a) Pihlaja, K.; Luoma, S. Acta Chem. Scand. 1968, 22, 2401. (b) Pihlaja, K., Äyräs, P. Acta Chem. Scand. 1970, 24, 204 and 531. (c) Pihlaja, K.; Kellie, G. M., Riddell, F. J. Chem. Soc. B 1972, 252. (d) Anteunis, M. J. O., Tavernier, D., Borremans, F. Heterocycles 1976, 4, 293 and references therein.

2. Eliel, E. L., Nader, F. W. J. Org. Chem. 1970, 92, 584.

3. Pihlaja, K.; Kivimäki, M., Myllyniemi, A-M., Nurmi, T. J. Org. Chem. 1982, 47, 4688.

4. Pihlaja, K., Nurmi, T. Isr. J. Chem. 1980, 20, 160.

5. Pihlaja, K., Kleinpeter, E. Carbon-13 NMR Chemical Shifts in Structural and Stereochemical Analysis, pp 91-94, Wiley-VCH: New York, 1994.

6. Burkert, U. Tetrahedron 1979, 35, 691.

7. (a) Pihlaja, K.; Äyräs, P. Acta Chem. Scand. 1970, 24, 531; (b) Eliel, E. L.; Knoeber, M. 1966, 88, 5347 and 1968, 90, 3444.

8. Pihlaja, K; Kellie, G.M.; Riddell, F.G. J. Chem. Soc. B 1972, 252.

9. Pihlaja, K., Ketola, M. Suomen Kemistil. 1968, B 41, 299.

10. Pihlaja, K., Ketola, M. Acta Chem. Scand. 1969, 23, 715. 